\title{
Skin Lesion Detection Using Fuzzy Approach and Classification with $\mathrm{CNN}$
}

\author{
Prashengit Dhar \\ Cox's Bazar City College, Bangladesh \\ Email: nixon.dhar@gmail.com
}

\section{Sunanda Guha}

Missouri State University

Email:sg75s@missouristate.edu

Received: 02 December 2020; Accepted: 20 January 2021; Published: 08 February 2021

\begin{abstract}
Skin lesion detection at early stage is very effective for patients. As a result, patients can get time for treatment. Moreover, this early detection helps a patient in the long-time survival. However, skin lesion detection from a dermoscopic images is not a general task. Due to inter and intra-observer variations in human interpretations, research on skin lesion detection from dermoscopic images become important. In this paper, we proposed a method to segment and detect lesion of skin from images. The proposed method is based on a set of rules of fuzzy logic approach. Firstly, a set of rules is applied on dermoscopic images. The output is then thresholded. Closing operation as a morphological tool is used on the thresholded image. Then area filtering takes a place which results in the desired output. With respect to different learning models, CNN shows better performance in classifying ISIC and Dermis-dermquest dataset. The system delivers a significant performance, which is remarkable and comparable.
\end{abstract}

Index Terms: Dermoscopic image, fuzzy logic, segmentation, lesion detection

\section{Introduction}

The number of skin cancer affected people is increasing day by day. Skin lesion detection from images is very challenging due to its complexity. Structure of lesion on skin also varies. Early detection of skin lesion can prevent one patient from skin cancer. One of the most common malignancy is Malignant melanoma. In USA, Malignant melanoma is known as deadly type of skin cancer and it is growing rapidly around the world. So skin lesion segmention is a very important issue.Moreover it is not easy to segment. Fuzzy logic rule based segemnation provides better way in segmenting images. A fuzzy rule-based approach to segment skin lesion from dermoscopic image is presented through this paper and also achieved a good classification rate.

Fuzzy logic generally deals with the data arising from cognition and computational perception like uncertainty, partially truth, impreciseness, or vagueness. Fuzzy Logic is a type of many-esteemed rationale in which reality estimations of factors might be any genuine number somewhere in the range of 0 and 1 where both are comprehensive. It is utilized to deal with the idea of incomplete truth, where reality worth may go between totally evident and totally false.[1] By differentiate, in Boolean rationale, reality estimations of factors may just be the number qualities 0 or 1 . Fuzzy logic is effective to develop decision making, pattern recognition identification, control and optimization based system The main concept is derived from analysis of dermoscopic images. The infected area i.e. lesion region is differed from general or healthy skin region. This difference leads to a better segmentation approach.Fuzzy logic permits the involvement of imprecise human calculations in various computing problems. In addition, it also provides useful meaning for multiple criteria with conflict resolution and improved assessment of possibilities. In 2009 , 68720 cases of skin lesion were calculated in all over the USA where 8441 people died [1]. Dermoscopy is a noninvasive technique of skin imaging. For the diagnosis of skin lesion, it becomes a guiding tool in recent years. It has optical based magnification for the interested region. As a result, subsurface becomes much visible than regular microscopic images [2]. But there is an issue that dermoscopy may decrease the diagnostic precision in case of inexperienced dermatologists [3]. In lieu of overcoming these diagnostic errors new computerized tools for image understanding is necessary. Incidents' complexity and visual interpretation's subjectivity causes these errors [4,5]. Multiple lesion object detection will be focused in future. 
This paper is ordered as follows: section III declares overview of the system, section IV covers discussion about dataset, brief details of lesion segmentation and detection are detailed in section V, section VI shows the feature and training whereas conclusion is demonstrated in section VII.

\section{Literature Review}

Segmentation allows evaluating diagnosis parameter like lesion diameter, bordering irregularities etc. Segmentation is a crucial phase in skin lesion detection, basically in computer aided diagnosis system (CAD). The use of deep learning is growing heavily. Few Deep learning models like- multitask CNN are used to segment skin lesion in trending years [6]. It includes lesion segmentation and independent binary classification. A CNN based segmentation named a 'SkinNet' is proposed by Vesal to segment lesion on skin from dermoscopic images [7]. In skin-net, dilated convolutions are applied at the down layers of encoder-branch, which in turn provides global context in extracted features. This is the moderated version of U-net [8]. Ren has offed a Faster-RCNN approach which is multi-tasking learning to localize lesion [9]. Few algorithms were also developed to detect border in dermoscopic images. Such algorithms proposed by Carlotto which involves in adaptive thresholding [10], median cut/ principal component transform (PCT) algorithm [11]. In addition, split and merge segmentation technique [12], $\mathrm{k}$ means clustering [13] are also included. A Color clustering-based algorithm is proposed by Schimid [14]. It uses CIE color space. The method smoothed the calculated histogram firstly. A perceptron classifier is used to obtain initial cluster center. Finally modified c means clustering algorithm segments the skin lesion image. Abbas and Baidaa proposed a system consists of two phases of several stages[17]. First phase checks for malignant and benign cancer type and last phase recognizes malignant melanoma type. Color,Texture Features and Geometry are used as feature and Neural networks as classifier.

By analyzing digital dermoscopy images, Xie proposed a method to classify tumors as malignant or benign[20]. It is three step approach. Lesions are extracted first with a Self generating Neural Networks (SGNN). Texture, border feature and color features are collected secondly. Network ensemble classifier classifies lesion object. A combination of Fisher vector (FV), deep convolutional neural network (CNN) and linear Support Vector Machine (SVM) is resented by Yu to assess dermoscopy image[21].

\section{Methodology}

This paper represents a framework for skin lesion segmentation and detection using fuzzy approach. Input image is filtered to remove noise. Fuzzy rules are then applied on the filtered image. Segmented image is appeared as outcome. The output image is then thresholded to get binary image. The pixel value of red, green, and blue components is used as fuzzy input. Closing as a morphological tool is applied to remove noise in binary image. Finally, area filtering is employed to get region of interest. Extracted ROI is then tested to classify. The proposed steps are shown in fig 1.

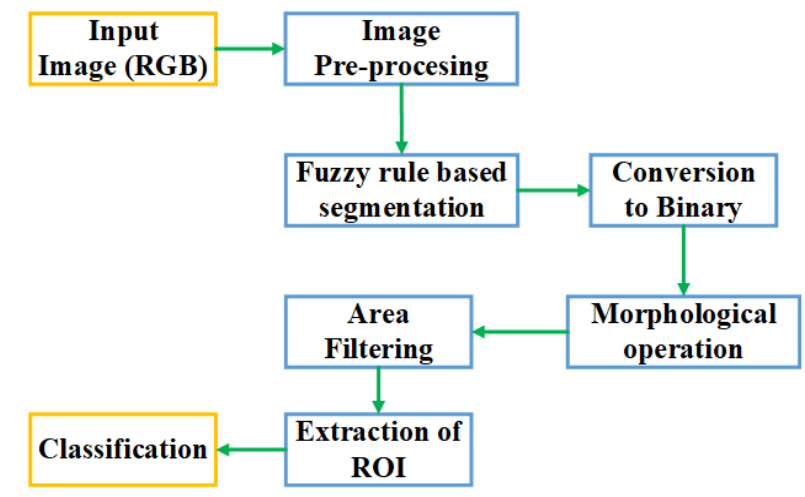

Fig. 1. Proposed detection system

\section{Dataset}

In image processing tasks, dataset is the mandatory part. It is better to have dataset on all possible situations. But testing on standard dataset is a good way. This study consider DermIs and DermQuest datasets[18,19]. Total 206 images exist in these datasets. These datasets are benchmark in skin lesion segmentation/skin cancer classification. Images in these datasets are divided as melanoma and non-melanoma. Melanoma is very popular and deathly type of skin cancer. ISIC skin cancer dataset[22] is also tested in classification for melanoma and pigmented Benign Keratosis classes. Fig. 2 shows the selected sample from dataset. 

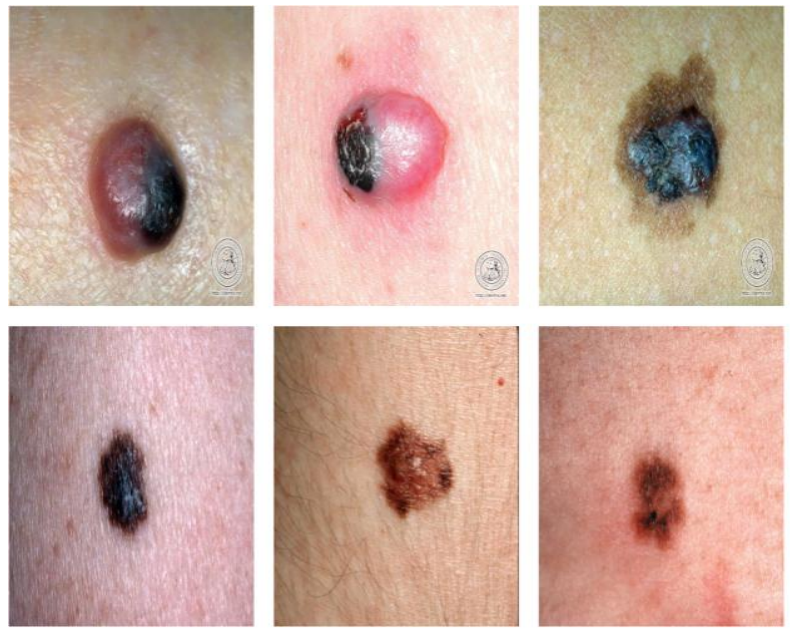

Fig. 2. Selected sample from dataset

\section{Segmentation}

Segmentation is crucial in the detection tasks. Segmentation comes after the pre-processing. Pre-processing makes the image finer to have a well segmented image. A set of fuzzy rules are applied on the pre-processed image. Fuzzy logic is very helpful in handling uncertainty lie in the images. An image may be affected by various parameters. Illumination of light is one of the most common uncertainties. Color of an image may get affected due to the light. Moreover, in case of shadow effect, fuzzy logic performs well. To overcome these types of uncertainties fuzzy logic is a well-known approach.

Rules are the most important thing in fuzzy logic approach. The rules re as follow:

i. $\quad$ r1 $=$ If red is red 1 and green is green 1 and blue is blue 1 then result is high

ii. r2 = If red is red2 then result is black

iii. $\mathrm{r} 3=$ If green is green 2 then result is black

iv. $\mathrm{r} 4=$ If blue is blue 2 then result is black

These four rules are used to segment image. The membership functions of fuzzy input (red, green, and blue) are derived by intuition. Fuzzy membership function is generalized from classical sets. fuzzy logic represents the degree of truth in a scale of value. Vague sets are defined by membership function in fuzzy. The input and output membership functions are shown in fig.3.
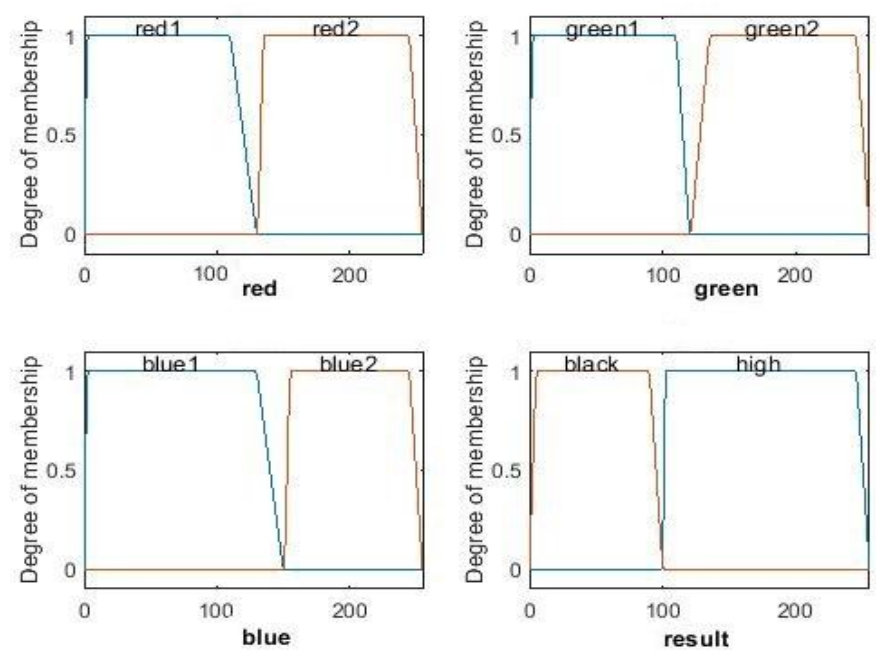

Fig. 3. Input and output variable of membership 
For each input there is a region which is not used. The regions are red2, green2 and blue2, respectively. If fuzzy interference system faced with these regions, system will provide output of black pixel. Input image is shown in fig.4 (a). Median filter is applied to remove noise from image. After applying these rules, segmented image is appeared as output in fig 4(b). The output image is then thresholded to convert in binary image which is shown in fig 4(c). Morphological closing operation is employed to fill missing boundaries and small gapes. Fig 4(d) is the output of morphological operation applied on fig 4(c). Moreover, it also helps in removing noise such as - spark. To get the required region (lesion region), area filtering is used. The region that contains maximum area is the desired region. It is shown in fig 4(e). Then using bounding box parameter, lesion area is cropped. Fig 4(f) is the desired ROI.

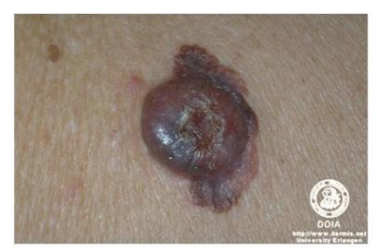

a)

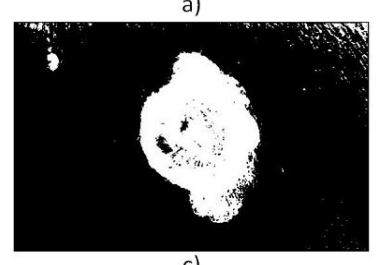

c)

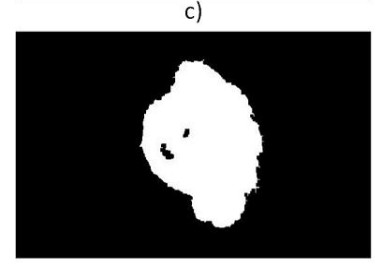

e)

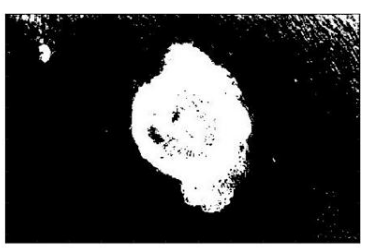

b)

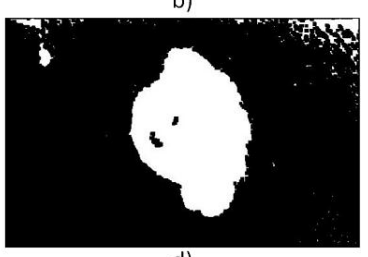

d)

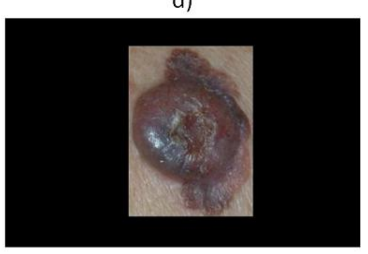

f)

Fig. 4. a) input image b) fuzzy processed image c) binarized image d) after closing operation e) area filtered f) extracted ROI

The trapezoidal membership function is used in all input and output as it fulfils our criteria, and it is better suited according to the segmentation. A large number of images were analyzed to set the rules. Fig. 5 and fig. 6 are also shows segmentation result.

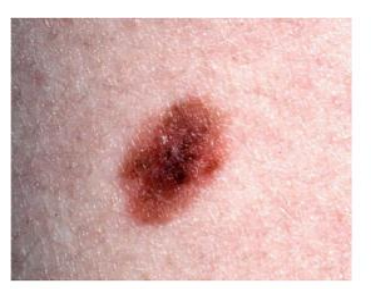

a)

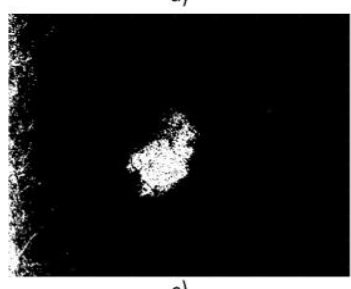

c)

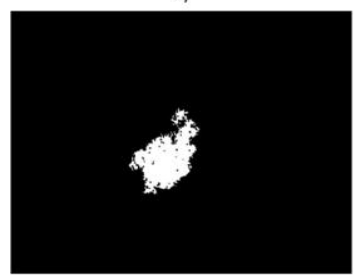

e)

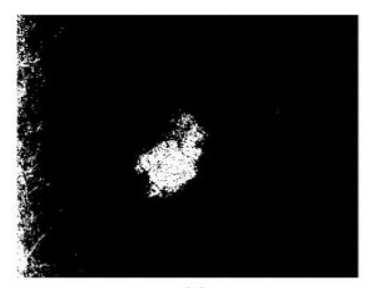

b)

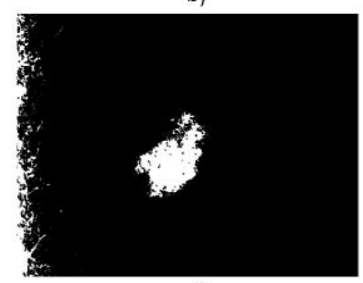

d)

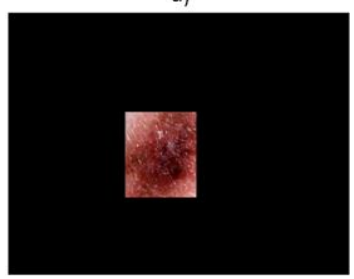

f)

Fig. 5. a) input image b) fuzzy processed image c) binarized image d) after closing operation e) area filtered f) extracted ROI 


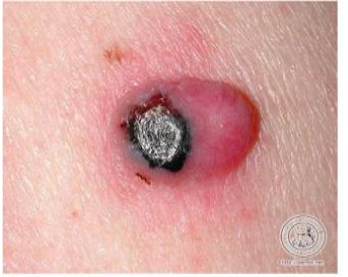

a)

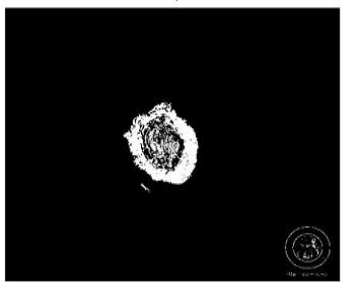

c)

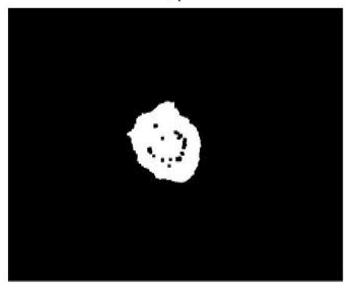

e)

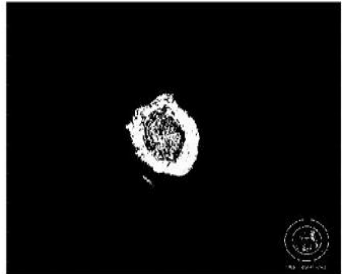

b)

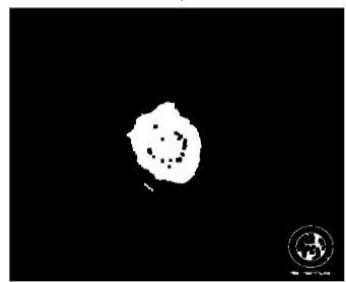

d)

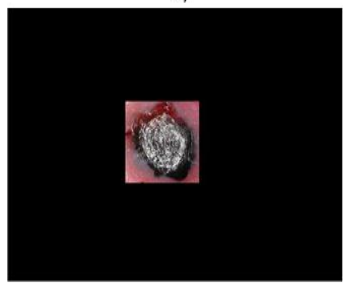

f)

Fig. 6. a) input image b) fuzzy processed image c) binarized image d) after closing operation e) area filtered f) extracted ROI

\section{Features and Training}

Convolutional Neural Network $(\mathrm{CNN})$ is employed in this study for feature extraction and classification. Moreover, CNN features at fully connected layers, are also trained using SVM. CNN is an artificial neural network, which is one of the most popular methods for analyzing images [15]. It has also been used to classify skin lesion [16].

CNN can detect pattern from images and this pattern detection makes CNN very powerful in image analysis. To train CNN, labeled data are required. Thus, it is called supervised learning. There are two components in CNN which are hidden layers and fully connected layers. The features get extracted in the hidden layers whereas fully connected layers are used to classify. Unlike other neural networks, CNN is not fully connected. This makes the training procedure simpler and causes lower model complexity.

The hidden layers of CNN are called convolutional layers. It may have other non-convolutional layers as well. Like other hidden layers, the convolutional layers transform the input and outputs the transformed input to the next layer. In each convolutional layer, the number of filters should be specified, and these filters are used to detect patterns. The filters can detect patterns like multiple edges, shapes, textures, objects etc. of the image. If a filter detects edges, then the filter is called edge detector. Some filters may detect corners, circles, squares etc. The deeper the network is, the more sophisticated the filters. The more sophisticated filters can detect more specific objects. The filters are represented by small matrix and the values of the matrix are random numbers. When the convolutional layer receives input, the filter slides over each filter sized set of pixels from the input itself until it slid over every filter sized block of pixels from the entire image. This sliding is called convolving. After the convolving of whole image, the layer outputs new representation of the initial input which is the dot product of entire filter matrix and the input. The next layer will receive this as its input. More complex filters are located deeper in the network.

Classification is applied on dermis- dermquest dataset for class label melanoma and non-melanoma. ISIC skin lesion dataset is also trained for melanoma and pigmented Benign Keratosis. Table 1 shows the skin lesion detection statistics. Different learning algorithms such as CNN, SVM, Adaboost and KNN have been applied on both datasets. Table 2 shows the accuracy of the learning algorithms for each of the dataset which shows that CNN proves better in both type of dataset classification. 
Table 1. Detection Statistics

\begin{tabular}{|c|c|c|}
\hline Type & $\begin{array}{c}\text { Number of } \\
\text { tested images }\end{array}$ & Detected lesion \\
\hline Images with lesion & 206 & 194 \\
\hline Images without lesion & 21 & 2 \\
\hline
\end{tabular}

Table 2. Accuracy of different algorithms in percentage

\begin{tabular}{|c|c|c|}
\hline Learning Algorithm & $\begin{array}{c}\text { Dermis- } \\
\text { dermquest }(\%)\end{array}$ & ISIC(\%) \\
\hline CNN & $\mathbf{8 5}$ & $\mathbf{9 4}$ \\
\hline SVM & 68 & 87.3 \\
\hline Adaboost & 66 & 88.3 \\
\hline KNN & 72 & 85.3 \\
\hline
\end{tabular}

Fig. 7 shows training and validation loss/accuracy for each epoch. Table 3 and 4 shows confusion matrix and classification report of CNN using Dermi sdermquest dataset whereas Table 5 and 6 shows confusion matrix and classification report of CNN using ISIC dataset.

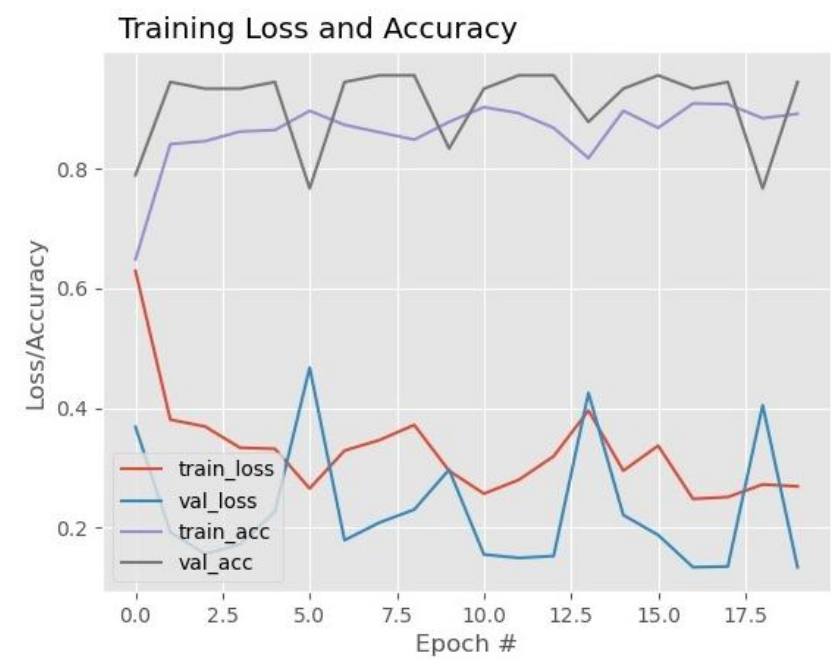

Fig 7. Dermis dermquest Training and validation loss and accuracy

Table 3. Confusion matrix of CNN using Dermis dermquest

\begin{tabular}{|c|c|c|}
\hline & melanoma & Non- melanoma \\
\hline melanoma & 11 & 0 \\
\hline Non- melanoma & 3 & 6 \\
\hline
\end{tabular}

Table 4. Classification report of CNN using Dermis dermquest

\begin{tabular}{|c|l|l|l|}
\hline & precision(\%) & recall(\%) & $\begin{array}{l}\text { f1- } \\
\text { score }(\%)\end{array}$ \\
\hline melanoma & 79 & 100 & 88 \\
\hline Non-melanoma & 100 & 67 & 80 \\
\hline \multicolumn{2}{|r|}{ Accuracy } & 85 \\
\hline \multicolumn{2}{|r|}{}
\end{tabular}

Table 5. Confusion matrix of CNN using ISIC

\begin{tabular}{|c|c|c|}
\hline & $\begin{array}{c}\text { Pigmented } \\
\text { Benign } \\
\text { Keratosis }\end{array}$ & Melanoma \\
\hline Pigmented Benign Keratosis & 44 & 3 \\
\hline melanoma & 2 & 41 \\
\hline
\end{tabular}


Table 6. classification report of CNN using ISIC

\begin{tabular}{|c|l|l|l|}
\hline & $\begin{array}{l}\text { precision } \\
(\%)\end{array}$ & $\begin{array}{l}\text { Recall } \\
(\%)\end{array}$ & $\begin{array}{l}\text { f1-score } \\
(\%)\end{array}$ \\
\hline $\begin{array}{c}\text { Pigmented Benign } \\
\text { Keratosis }\end{array}$ & 96 & 94 & 95 \\
\hline melanoma & 93 & 95 & 94 \\
\hline \multicolumn{2}{|r|}{ Accuracy } & 95 \\
\hline
\end{tabular}

\section{Conclution}

This paper proposes a skin lesion detection system using fuzzy rule-based approach and classification with CNN. Segmentation of lesion is made by following fuzzy rules. Fuzzy rule-based method is useful to get sharp segmentation. Fuzzy Logic technique emphasizes on what the system would do rather than trying to model in what way it works. Fuzzy logic depends on the perception that individuals settle on choices dependent on uncertain and non-numerical data. Fluffy models or sets are numerical methods for speaking to ambiguity and loose data. These models have the capacity of perceiving, speaking to, controlling, deciphering, and using information and data that are obscure and need conviction. Detection is tested on one public dataset-dermis dermquest and the classification is tested on two public datasets. Outcomes are significant in localizing lesion. Moreover classification result is also acceptable. CNN performs well in classifying images of skin lesion. This framework will be much useful in detecting skin lesion as well as localizing lesion. Later effort will be given to update this system to detect and classify mult0le lesion objects precisely and to develop other algorithm that can have higher efficiency.

\section{References}

[1] Jemal A, Siegel R, Ward E, Hao Y, Xu J, Murray T, et al. Cancer statistics. A Cancer Journal for Clinicians 2008; 59:225-49. CA.

[2] Argenziano G, Soyer HP, De Giorgi V. Dermoscopy: a tutorial. EDRA Medical Publishing \& New Media; 2002.

[3] Binder M, Schwarz M, Winkler A, Steiner A, Kaider A, Wolff K, et al. Microscopy:a useful tool for the diagnosis of pigmented skin lesions for formally trained dermatologists. Archives Of Dermatology 1995;131(3):286-91.

[4] Fleming MG, Steger C, Zhang J, Gao J, Cognetta AB, Pollak I, et al. Techniques for a structural analysis of dermatoscopic imagery. Computerized Medical Imaging and Graphics 1998.

[5] Celebi ME, Aslandogan YA, Stoecker WV, Iyatomi H, Oka H, Chen X. Unsupervised border detection in dermoscopy images. Skin Research and Technology 2007;13(4):454-62.

[6] Yang, X., Zeng, Z., Yeo, S.Y., Tan, C., Tey, H.L., Su, Y.: A novel multi-task deep learning model for skin lesion segmentation and classication. arXiv preprint arXiv:1703.01025 (2017)

[7] Vesal, S., Ravikumar, N., Maier, A.: Skinnet: A deep learning framework for skin lesion segmentation. (2018) preprint,

[8] Ronneberger, O., Fischer, P., Brox, T.: U-net: Convolutional networks for biomedical image segmentation. In Navab, N., Hornegger, J., Wells, W.M., Frangi, A.F.,eds.: Medical Image Computing and Computer-Assisted Intervention \{ MICCAI 2015, Cham, Springer International Publishing (2015) 234-241

[9] Ren, S., He, K., Girshick, R., Sun, J.: Faster r-cnn: Towards real-time object detection with region proposal networks. In: Advances in neural information processing systems. (2015) 91-99

[10] Carlotto MJ. Histogram analysis using a scale-space approach. IEEE Transaction on Pattern Analysis and Machine Intelligence 1987;1(9):121-9.

[11] Hance GA, Umbaugh SE, Moss RH, Stoecker WV. Unsupervised color image segmentation with application to skin tumor borders. IEEE Engineering in Medicine and Biology 1996;15(1):104-11.

[12] Dhawan AP, Sicsu A. Segmentation of images of skin lesions using color and texture information of surface pigmentation. Computerized Medical Imaging and Graphics 1992;16(3):163-77.

[13] Gao J, Zhang J, Fleming MG, Pollak I, Cognetta AB. Segmentation of dermatoscopic images by stabilized inverse diffusion equations. Proceedings of the IEEE International Conference Image Process 1998; 3:823-7.

[14] Schmid P. Segmentation of digitized dermoscopic images by two-dimensional color clustering. IEEE Transaction on Medical Imaging 1999; 18:164-71.

[15] LeCun Y, Bengio Y, Hinton G. Deep learning. Nature 2015 May 28;521(7553):436-444

[16] Brinker, Titus Josef, et al. "Skin cancer classification using convolutional neural networks: systematic review." Journal of medical Internet research 20.10 (2018): e11936.

[17] Abbas Hanon. Alasadi, Baidaa M.ALsafy,"Early Detection and Classification of Melanoma Skin Cancer", International Journal of Information Technology and Computer Science(IJITCS), vol.7, no.12, pp.67-74, 2015. DOI: 10.5815/ijitcs.2015.12.08

[18] https://www.dermis.net/dermisroot/en/home/index.htm

[19] https://uwaterloo.ca/vision-image-processing-lab/sites/ca.vision-image-processing-lab/files/uploads/files/skin_image_data_set2.zip

[20] Xie F, Fan H, Li Y, Jiang Z, et al (2017). Melanoma classification on dermoscopy images using a neural network ensemble model. IEEE Trans Med Imaging, 36, 847-58.

[21] Yu Z, Ni D, Chen S, Qin J, et al (2017). Hybrid dermoscopy image classification framework based on deep convolutional neural network and fisher vector. IEEE 14th International Symposium on Biomedical Imaging, pp 301-4.

[22] https://www.kaggle.com/c/siim-isic-melanoma-classification/data 


\section{Authors' Profiles}

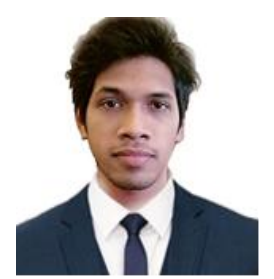

Prashengit Dhar received his B.Sc. degree in Computer Science and Engineering from University of Science and Technology Chittagong (USTC) and M.Sc. degree in Computer Science and Engineering from Port City International University. Currently he is working as a lecturer in a college. He has published many papers in conference and journal. His research interests include image processing, pattern recognition and machine learning.

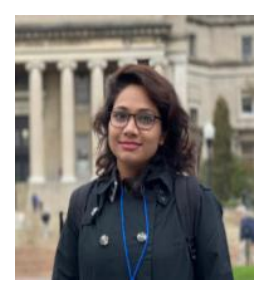

Sunanda Guha received her B.Sc. and M.Sc. degree in Computer Science and Engineering from University of Chittagong. Currently she is studying her Masters in computer science in the Missouri State University. She has published several papers in conference and journal. Her research interests include Machine Learning, Expert Systems, Internet of Things, Big Data and Image processing.

How to cite this paper: Prashengit Dhar, Sunanda Guha, " Skin Lesion Detection Using Fuzzy Approach and Classification with CNN ", International Journal of Engineering and Manufacturing (IJEM), Vol.11, No.1, pp. 11-18, 2021. DOI: 10.5815/ijem.2021.01.02 